ARTICLE

\title{
Replication dynamics of recombination-dependent replication forks
}

Karel Naiman (10 ${ }^{1 凶}$, Eduard Campillo-Funollet (10 ${ }^{1}$, Adam T. Watson ${ }^{1}$, Alice Budden (D) ${ }^{1}$, Izumi Miyabe ${ }^{1}$ \& Antony M. Carr (1D ${ }^{1 凶}$

Replication forks restarted by homologous recombination are error prone and replicate both strands semi-conservatively using Pol $\delta$. Here, we use polymerase usage sequencing to visualize in vivo replication dynamics of HR-restarted forks at an S. pombe replication barrier, RTS1, and model replication by Monte Carlo simulation. We show that HR-restarted forks synthesise both strands with Pol $\delta$ for up to $30 \mathrm{~kb}$ without maturing to a $\delta / \varepsilon$ configuration and that $\mathrm{Pol} \alpha$ is not used significantly on either strand, suggesting the lagging strand template remains as a gap that is filled in by Pol $\delta$ later. We further demonstrate that HRrestarted forks progress uninterrupted through a fork barrier that arrests canonical forks. Finally, by manipulating lagging strand resection during HR-restart by deleting pku70, we show that the leading strand initiates replication at the same position, signifying the stability of the $3^{\prime}$ single strand in the context of increased resection.

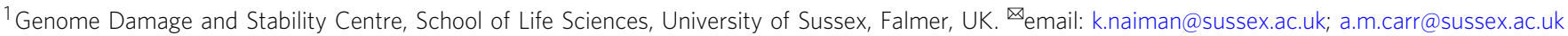


E ach human cell has to correctly copy $\sim 6.5$ billion bps when it divides. DNA replication is impressively accurate such that each cell division produces only a handful of errors ${ }^{1}$. However, numerous internal and external factors can impact on replication fidelity and cancer development is associated with increased intrinsic replication stress ${ }^{2}$. A number of other diseases including neurological, neurodegenerative and neuromuscular disorders (e.g. Friedreich's ataxia, myotonic dystrophy, spinocerebrallar ataxias, fragile X syndrome and Huntington's disease) are caused by replication-dependent slippage triggering nucleotide repeats instability ${ }^{3}$.

Replication forks (RFs) are disrupted by different obstacles ${ }^{4}$, including DNA-bound proteins, DNA damage, structure forming sequences (i.e. G-quadruplexes, i-motifs, inverted repeats, trinucleotide repeats) and DNA/RNA hybrids. An RF encountering such a barrier might be resolved by one of the following three strategies: (i) activation of the intra-S-phase checkpoint to stabilise the arrested RF. This preserves the replisome and the fork structure to protect the DNA from inappropriate modification. A stabilised RF can resume replication when the blockage is resolved, for example by the activity of DNA repair or accessory helicases. Alternatively, a stabilised fork can be resolved by an incoming replication fork (termination). However, not all arrested forks expose sufficient single-stranded DNA to activate the intra-S phase checkpoint and such are said to collapse. (ii) A collapsed fork can also be rescued by a converging RF. This strategy is efficient due to an excess of potential replication origins (dormant origins) that can be activated upon replication stress $^{5}$. However, some fragile sites have a paucity of origin activity over megabase distances ${ }^{6}$. These regions are particularly sensitive to replication problems, likely because two converging forks that are forced to travel long distances are more likely to both collapse and there are no dormant origins present in the intervening region. (iii) To overcome the consequences of two converging forks collapsing, or the collapse of single forks in regions of unidirectional replication such as telomeres, cells have developed mechanisms to restart a collapsed RF by homologous recombination $(\mathrm{HR})^{7}$.

The major pathway of HR-mediated fork restart requires Rad51 and the classical HR mediators. Restart can occur from a one-ended DNA double strand break (DSB), such as would occur if the RF encounters a nick ${ }^{8}$, or from a resected replication fork, likely following fork reversal that generates a 4-way DNA junction known as a chicken-foot ${ }^{9}$. To explore the latter mechanism of HR-restart of collapsed RFs we developed a model system in fission yeast using a unidirectional replication fork barrier, RTS1. Using this system, we and others have demonstrated that HR-dependent restart occurs rapidly $(\sim 18$ $\left.\min ^{10}\right)$, does not require a DSB intermediate ${ }^{11}$ and likely involves an initial fork reversal step that allows regulation of resection via $\mathrm{Ku}$ binding and subsequent displacement by the Mre11-Rad50-Nbs1 complex ${ }^{12}$. We and others have also shown that the restart of arrested forks is associated with various types of genetic instability: ectopic recombination events can restart the fork at the wrong locus causing rearrangements such as translocations ${ }^{11}$, while forks restarted at the correct locus are intrinsically more error prone than a canonical fork. The errors intrinsic to the restarted replication machine include increased replication slippage ${ }^{13}$, recombination between direct repeats ${ }^{14}$ and the coupled formation of dicentric and acentric isochromosomes at inverted repeats ${ }^{15}$. The increase in error frequency implies that HR-restarted RFs are different from the canonical RF. Indeed, we found that, unlike canonical replication (where polymerase Pol $\varepsilon$ synthesises the leading strand and Pol $\delta$ the lagging strand), both the leading and lagging strands are synthesised by Pol $\delta$ despite the fact that replication remains semi-conservative ${ }^{10}$ and does not therefore progress as a migrating D-loop as is seen during break-induced replication ${ }^{16}$.

One limitation of current assays to characterise fork restart at RTS1 is the inability to track replication dynamics around the site of the restarted fork. Thus, many conclusions are drawn from assays that measure ectopic recombination, the mutagenic potential of the restarted RF or which quantify specific DNA structures using 2D gels. Here we combine polymerase usage sequencing $\left(\mathrm{Pu}-\mathrm{seq}^{17}\right)$ with a Monte Carlo model of $S$. pombe DNA replication ${ }^{18}$ to complement these assays. $\mathrm{Pu}$-seq relies on mutant replicative polymerases that introduce an increased frequency of ribonucleotides during DNA synthesis ${ }^{19}$. By mapping the strand-specific location of ribonucleotides across the genome and comparing the profiles of strains mutated in the different replicative polymerases, $\mathrm{Pu}$-seq tracks polymerase usage across the genome. Pu-seq thus allows the direct visualisation of restart in vivo and is complementary to the above mentioned indirect indicators such as mutagenic outcomes. By modelling $\mathrm{Pu}$-seq derived replication dynamics and comparing this with experimental data we are able to gain significant insights into the process of HR-dependent replication restart.

Consistent with our previous work ${ }^{10}$, we confirm that Pol $\delta$ synthesises both the leading and lagging strands (referred to as $\delta /$ $\delta$ configuration) and demonstrate that the HR-restarted replication machine does not mature into an $\varepsilon / \delta$ configuration over $\sim 30$ $\mathrm{kb}$, but continues in the $\delta / \delta$ configuration until it is terminated upon encountering a converging fork. We also provide direct evidence that Pol $a$ does not participate significantly in the DNA synthesis mediated by the HR-restarted RF. This suggests that the lagging strand template remains as a gap that is filled-in following fork merger.

During fork restart the lagging strand is degraded and, following Rad51-dependent strand invasion, the leading strand acts as the template to initiate replication. To explore the stability of the leading stand we manipulated the timing of restart and the extent of resection behind the fork arrested at RTS1 by deleting $p k u 70^{12}$. We confirm that extending resection of the lagging strand impacts on the timing of HR-restart but does not influence site of HR-restarted fork initiation. This suggests that leading strand $3^{\prime}$ end stability is not influenced by $\mathrm{Ku}$ or $5^{\prime}$ end resection.

\section{Results}

Replication forks are efficiently arrested at RTS1, a programmed unidirectional replication fork barrier of $\sim 850 \mathrm{bp}$ DNA derived from the mating type locus of fission yeast ${ }^{20}$. RTS 1 itself is not intrinsically difficult to replicate and is only active as a fork barrier when bound by Rtf1. To maximise the distance that a restarted fork travels we tested four locations, introducing the RTS1 sequence immediately adjacent to an efficient early firing origin that boarders a transition zone between early and late replicating regions ${ }^{17}$ and performed $\mathrm{Pu}$-seq on each in the presence of RTS1 barrier activity (Supplementary Fig. 1). From the resulting Pu-seq traces we chose a locus on chromosome II, which showed the highest extent of HR-restarted replication over distance. At this locus (Fig. 1a), when the left-to-right RF emerges from the early origin and reaches RTS1, it is arrested and subsequently restarted by HR. During this time, the distant $(\sim 30 \mathrm{~kb}$ away) late origin is activated, but the difference in timing of origin activation and the time taken for fork movement means that the converging right-to-left moving fork does not reach the RTS1 sequence and the HR-restarted RF travels left-to-right for $\sim 5-15 \mathrm{~kb}$, until it terminates with the convergent right-left fork.

To further delay the converging fork, we next inserted 10x copies of the Ter2-Ter3 sequence ${ }^{21}$ from the $S$. pombe ribosomal DNA replication fork barrier (rRFB) $12 \mathrm{~kb}$ downstream of RTS1, 
a
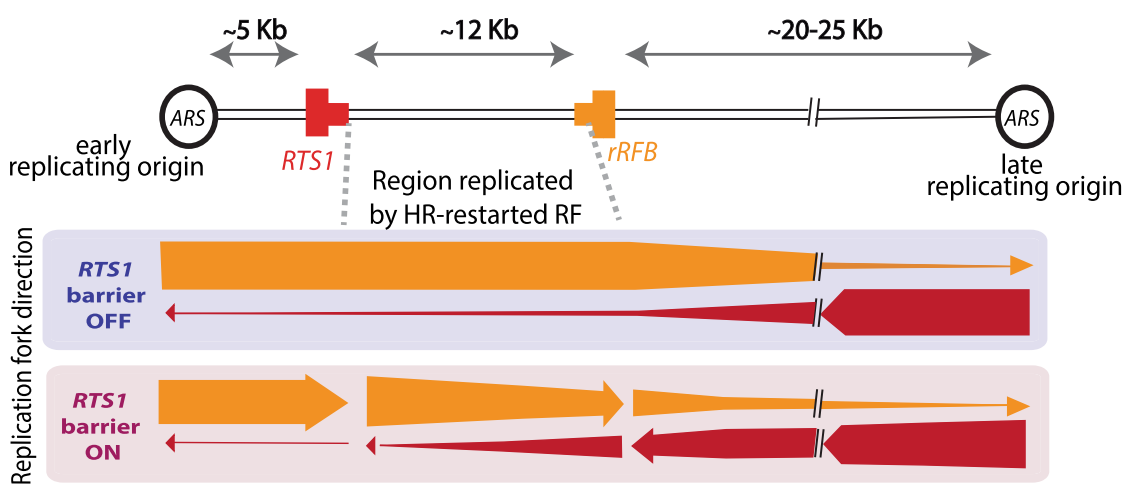

b
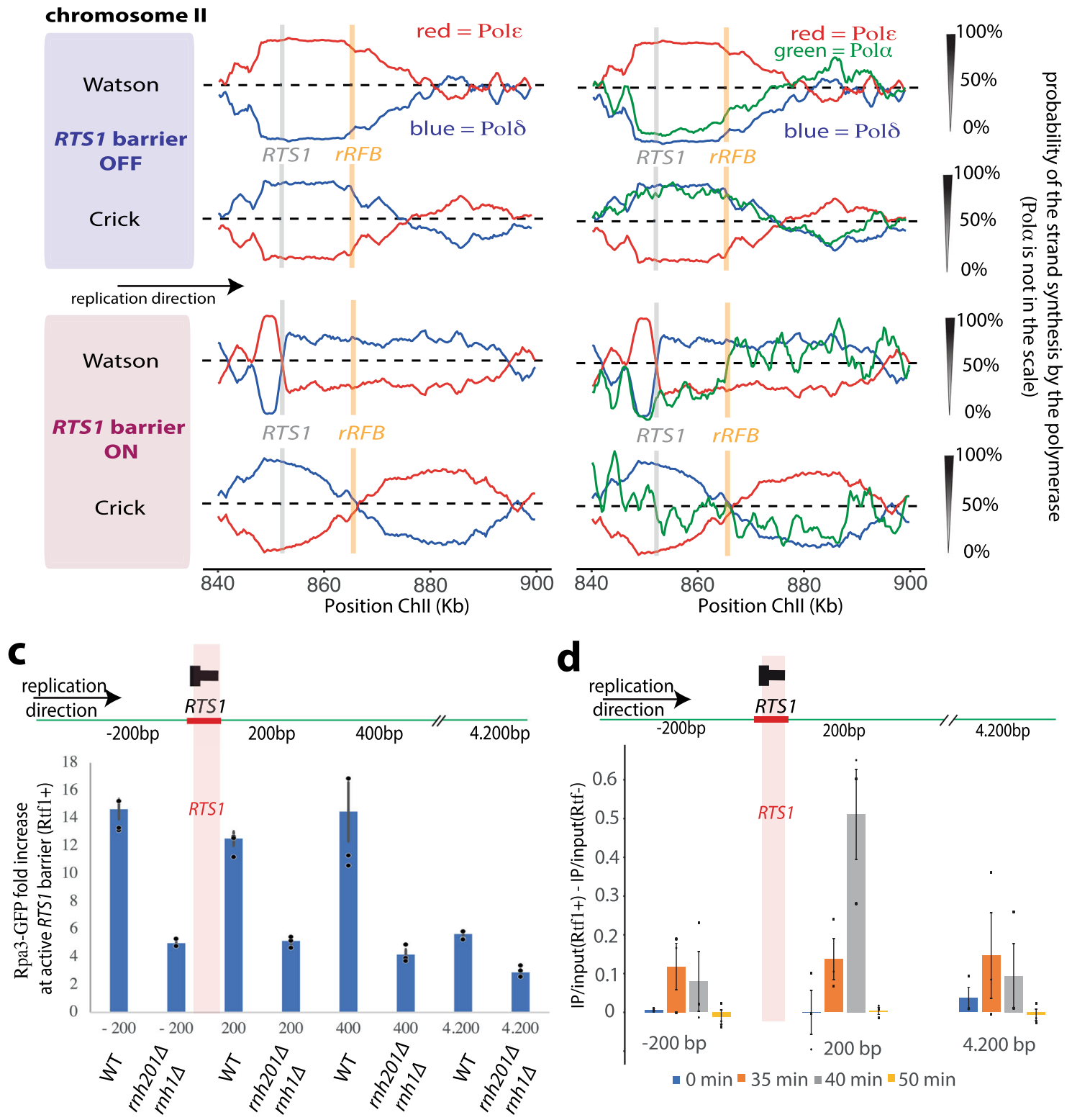

between RTS1 and the late firing origin (Fig. 1a). At the rRFB barrier, RFs do not collapse ${ }^{22}$, likely because the rRFB-binding proteins activate the fork protection complex ${ }^{23}$, and the RF is only transiently paused and does not undergo HR restart. To establish the extent of the delay caused by the rDNA barrier, we

first inserted the 10x Ter2-Ter3 close to a strong origin (on ChrI) and used $\mathrm{Pu}$-seq to monitor fork direction across the locus. We then implemented a recently described Monte Carlo model of $S$. pombe DNA replication ${ }^{18}$ that we have modified to account for programmed replication barriers (see Materials and Methods 
Fig. 1 Polymerase usage following HR-restart. a Schematic of the RTS1-rRFB locus on chromosome II. The positions of the directional RTS1 and rRFB barriers are shown as red and orange, respectively. The thick bar represents the directionality of fork arrest. ARS autonomously replicating sequence. The direction (see panel $\mathbf{b}$ ) of unperturbed and perturbed replication at this locus is indicated by the thickness of arrows underneath. $\mathbf{b}$ Pu-seq traces of the Chrll locus. Top two traces: RTS1 barrier activity off (rtf1- $d$ ). Bottom two traces: RTS1 barrier activity on (rtf1+). Left: the usage of Pol $\delta$ (blue) and Pol $\varepsilon$ (red) are shown on the Watson and Crick strands. Note the switch from Pol $\varepsilon$ to Pol $\delta$ on the Watson strand at the RTS1 site is indicative of a change in polymerase usage on the leading strand when RTS1 barrier activity is on. Right: The same traces overlaid with data for Pol $\alpha$ (green). Pol $\alpha$ data is not to scale, see Methods section. c Chromatin immunoprecipitation of Rpa3-GFP at the indicated positions (relative to base 1 of the RTS1 sequence) in unsynchronised cells with either $r n h 1^{+}$rnh201+ (WT) or rnh1-d rnh201-d backgrounds. Data presented are relative to the ade $\sigma^{+}$control locus. $n=3$ biological repeats. Error bars: standard deviation of the mean. $\mathbf{d}$ Chromatin immunoprecipitation using the S9.6 antibody in wild-type cells synchronised in $\mathrm{G} 2$ and released into $\mathrm{S}$ phase. $n=3$ biological repeats. Error bars: standard deviation of the mean.

section). Using this programme, we modelled various delay scenarios. The best match to the experimental data was $\sim 6$-min delay for the rRFB construct (Supplementary Fig. 2).

Using our optimised construct for Pu-seq we observe that the majority of the $12 \mathrm{~kb}$ between the RTS 1 and rRFB is replicated by the HR-restarted fork when the barrier is active (Fig. 1a, b). In previous work using qPCR immediately upstream and downstream of the RTS sequence we have estimated the delay time at the RTS1 barrier to be $\sim 18 \mathrm{~min}^{10}$. By applying our modified model, we show that the best fit for the experimental data is a delay at RTS1 of $11 \mathrm{~min}$ (Supplementary Fig. 3). It should be noted, however, that we assume in the model that replication speed following restart is equivalent to canonical replication (we used $1.8 \mathrm{~kb} /$ minute). If this assumption was incorrect, the optimal delay time would change. For example, if we model HR restarted fork speed at $3 \mathrm{~kb}$, the optimal delay time from the model is 17.5 min; see discussion.

Polymerase alpha usage during HR-restarted replication. In our original description of $\mathrm{Pu}$-seq ${ }^{17}$ we used two strains for each experiment: one containing a mutation in the gene encoding the catalytic subunit of Pol $\delta$ (cdc6-L591G or cdc6-L591M) and one containing an equivalent mutation in the gene encoding the catalytic subunit of Pol $\varepsilon(c d c 20-M 630 F)$. To monitor the contribution of polymerase $\alpha$, we included an additional strain containing an equivalent mutation in the gene encoding the catalytic subunit of Pol a (swi7-L850F). Previously we suggested that Pol a contributed less to replication of the lagging strand by HR-restarted forks than for canonical forks because Pol a dependent mutagenesis was reduced $\sim 6$-fold when forks were replicated by HR-restarted replication ${ }^{10}$. From the Pu-seq traces (Fig. 1b; also see Supplementary Fig. 4) we now confirm and extend this: Pol a usage is significantly reduced during synthesis of the lagging strand when replication is performed by an HRrestarted fork.

This observation is consistent with a model whereby the HRrestarted fork synthesises the complete leading strand without coupled Okazaki fragment synthesis on the lagging strand. Presumably, upon termination, what would be the lagging strand is then replicated by Pol $\delta$ from the $3^{\prime}$ end derived from the converging forks leading strand. This would result in RPA-bound ssDNA accumulating significantly in the region and the possibility that RNA:DNA hybrids may also accumulate ${ }^{24}$. To establish if this was the case, we examined the accumulation of RPA by ChIP at several sites around the locus in asynchronous $r t f 1^{+}$cells $(\sim 10 \%$ of which will be in S phase; Fig. 1c). We observed significant RPA accumulation at that part of the locus replicated by an HR-restarted fork when normalised against a locus (ade6) that was replicated by canonical forks. This was attenuated in an rnh201-d rnh1-d double mutant background, suggesting competition between RPA and RNA:DNA hybrids, as previously reported at resected double strand breaks ${ }^{24}$. Using an antibody with specificity to RNA:DNA hybrids we also saw evidence for the accumulation of RNA:DNA hybrids in S phase (Fig. 1d).

HR-restarted forks do not mature to an $\boldsymbol{\varepsilon} / \boldsymbol{\delta}$ configuration. We had previously reported that HR-dependent replication is semiconservative and proceeds in a manner by which Pol $\delta$ synthesises both the leading and lagging strand ( $\delta / \delta$ configuration). We did not see any evidence that HR-dependent replication reverted to a more canonical $\varepsilon / \delta$ configuration over $2-3 \mathrm{~kb}^{10}$. The data in Fig. 1b extend this: the fact that there is no increased Pol $\varepsilon$ usage between the RTS1 and rRFB sites $(\sim 12 \mathrm{~kb})$ on the leading (Watson) strand implies there is no maturation of the HRrestarted replication machine to the canonical distribution of polymerase labour.

To explore this further we designed an additional construct where a second RTS1 sequence was integrated in the opposing orientation to the first, and close to the late origin (Fig. $2 \mathrm{a}$ ). This second RTS1 sequence was intended to arrest the right-to-left fork emerging from the late origin and promote its HR-restart. The rationale was to isolate an origin-free region of $\sim 30 \mathrm{~kb}$ and force this to be replicated by HR-restarted forks. If HR-restarted forks do transition from $\delta / \delta$ configuration to $\varepsilon / \delta$, then we would predict significant evidence of $\mathrm{Pol} \varepsilon$ usage in the $\mathrm{Pu}$-seq traces. As is evident (Fig. 2b) we see no significant Pol $\varepsilon$ usage on either strand across $>30 \mathrm{~kb}$, commensurate with HR-restarted forks replicating consistently in the $\delta / \delta$ configuration. It is notable that no significant dormant origin firing is evident in the region between the two RTS1 sequences. Thus, without HR-mediated fork restart, this region would remain unreplicated, suggesting that this mechanism has evolved specifically to rescue replication when dormant origins are not available.

HR-restarted forks are insensitive to RTS1. In previous work using 2D gels we estimated that replication arrest at RTS1 was $>90 \%$ efficient ${ }^{11}$. However, in the traces shown in Fig. $1 \mathrm{~b}$ only $\sim 70 \%$ of forks appeared to switch replication from an $\varepsilon / \delta$ to a $\delta / \delta$ configuration. We reasoned that this may reflect the fact that, in these experiments, we are relying on endogenous $r t f 1^{+}$levels while in previous work we regulated the expression of $r t f 1^{+}$ through use of an inducible $n m t 1$ promoter. To test the possibility that the level of $r t f 1^{+}$transcription influences the efficiency of arrest at $R T S 1$, we replaced the endogenous $r t f 1^{+}$promoter with the $a d h 1^{+}$promotor (adh- $\left.r t f 1^{+}\right)$and performed an additional $\mathrm{Pu}$-seq experiment (Fig. 3a). When compared to $r t f 1^{+}$, the adh$r t f 1^{+}$traces indeed showed an increased transition from the $\varepsilon / \delta$ to the $\delta / \delta$ configuration. Using our model of DNA replication, we estimated that the efficiency of RTS1 barrier in arresting a canonical fork in the strain with endogenous $r t f 1^{+}$reached $\sim 70 \%$ but in adh-rtf1 $1^{+}$, the blocking capacity of RTS1 increased to $90 \%$ (Supplementary Fig. 5). We thus conclude that the RTS1 barrier is indeed highly efficient when Rtf1 is not limiting.

The fact that the $r t f 1^{+}$experiment only arrests $\sim 70 \%$ of forks prompted us to address a longstanding question: does the HR- 
a

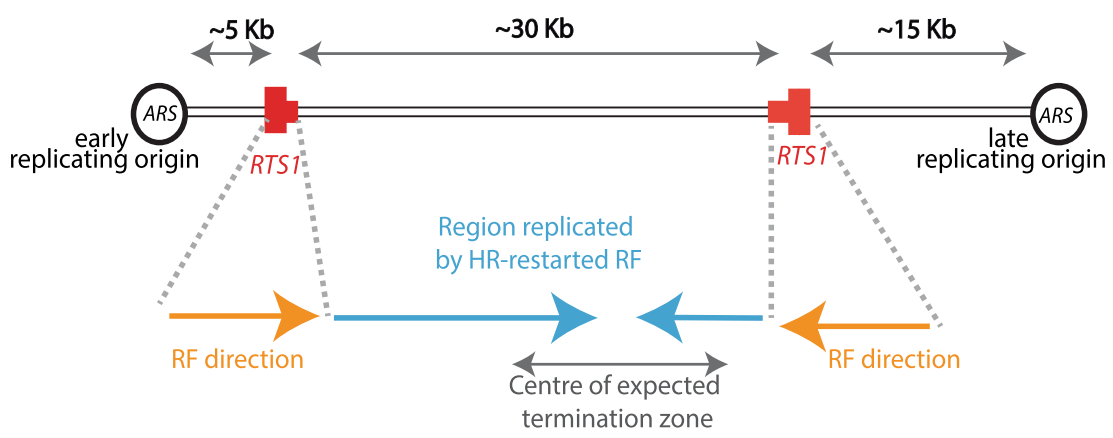

$\mathrm{RF}=$ replication fork

b

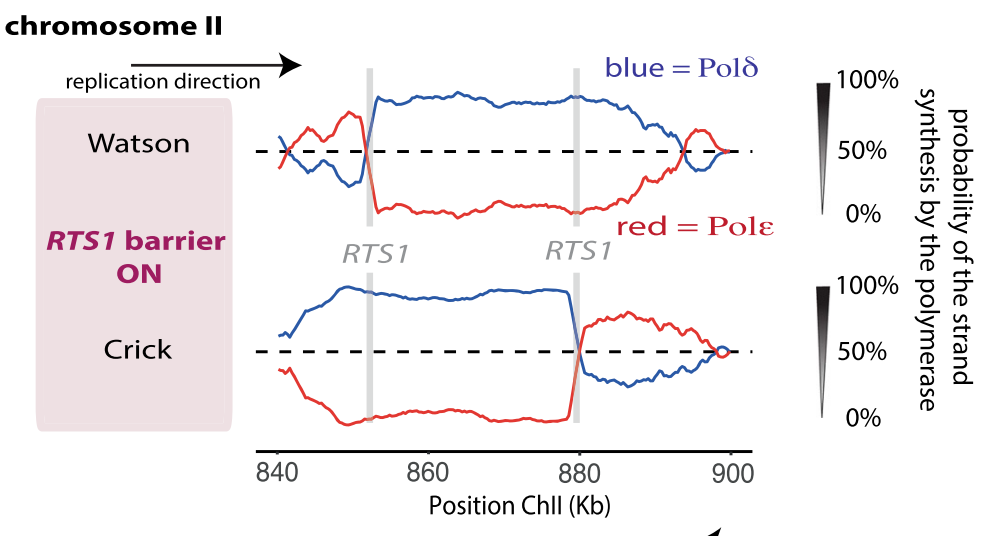

replication direction

Fig. 2 HR-restarted replication does not mature from the $\boldsymbol{\delta} / \boldsymbol{\delta}$ to $\boldsymbol{\varepsilon} / \boldsymbol{\delta}$ configuration. a Schematic of the inverted RTS1 locus on chromosome II. The positions of the directional RTS1 barriers are shown in red, with the thick bar indicating the directionality of fork arrest. $\mathbf{b}$ Pu-seq traces from the modified locus when the barrier is active (adh-rtf ${ }^{+}$). Note: the transition from Pol $\varepsilon$ (red) to Pol $\delta$ (blue) on the Watson strand at the left-side RTS1 site and the transition from Pol $\varepsilon$ to Pol $\delta$ on the Crick strand at right-side RTS1 site.

restarted fork also get arrested at an RTF1 barrier, or is it intrinsically resistant to this particular barrier? If the latter were the case, it would explain why the HR-restarted fork can overcome the initial barrier, which stopped the canonical fork. We therefore modified the locus to include a second copy of RTS1 $\sim 1.8 \mathrm{~kb}$ (Fig. 3b) downstream and performed additional Pu-seq experiments with and without the presence of $r t f 1^{+}$(Fig. 3c). In the absence of fork arrest the traces of polymerase usage are as expected, with the vast majority of forks travelling left-right. In the presence of $r t f 1^{+}$, again as expected, $\sim 70 \%$ of left-right forks arrest at the first RTS1 sequence and restart in a $\delta / \delta$ configuration. This is indicated by a sharp transition in Pol $\delta$ usage on the Watson strand at the first RTS1 site. At the second RTS1 sequence, the majority of the remaining $30 \%$ of canonical forks that were not arrested at the first RTS1 barrier arrest and restart at the second RTS1 site. This is indicated by the additional Pol $\delta$ usage inflection on the Watson strand at the second RTS1 site.

We can thus evaluate if the $70 \%$ of HR-restarted forks that were arrested at the first barrier are significantly delayed as they pass through the second barrier by comparing the position of terminations (fork merger events). This can be seen from the position and gradient of $\mathrm{Pol} \varepsilon$ usage on the Crick strand. In this context Fig. $3 c$ demonstrates two things: first, activating either one or two barriers significantly shifts the position of the termination events to the left, commensurate with a delay to leftright replication forks. Second, when the barrier(s) are active, termination positions and frequencies do not change significantly between the situations where there is either one or two barriers. Thus, we can conclude that the second RTS1 barrier does not significantly delay the progress of HR-restarted replication forks. Again, we can apply our model to the scenario, applying different delays to forks restarted at the first barrier when they pass through the second barrier (Supplementary Fig. 6). The best fit to the experimental data is no delay of HR-restarted forks at the second barrier. We thus propose that HR-restarted forks are unaffected by the RTS1 barrier.

Altering upstream resection of the lagging strand. A role for $\mathrm{Ku}$ in restricting $5^{\prime}$ end resection behind RTS1 arrested forks was recently reported ${ }^{12}$. In the absence of $p k u 70$, resection was increased by a factor of $>2$, to beyond $1800 \mathrm{bp}$ and replication restart was modestly delayed. To address the consequences of $\mathrm{Ku}$ loss on replication dynamics we introduced a $p k u 70$ deletion mutation into our Pu-seq strains. We also modified the Pu-seq protocol by introducing a rnh201-RED mutation (RED; ribonuclease excision defective) instead of the rnh201 deletion. RNase $\mathrm{H} 2$ can remove single ribose bases in DNA through initiating the ribonucleotide excision repair (RER) pathway ${ }^{25}$. It can also 
a

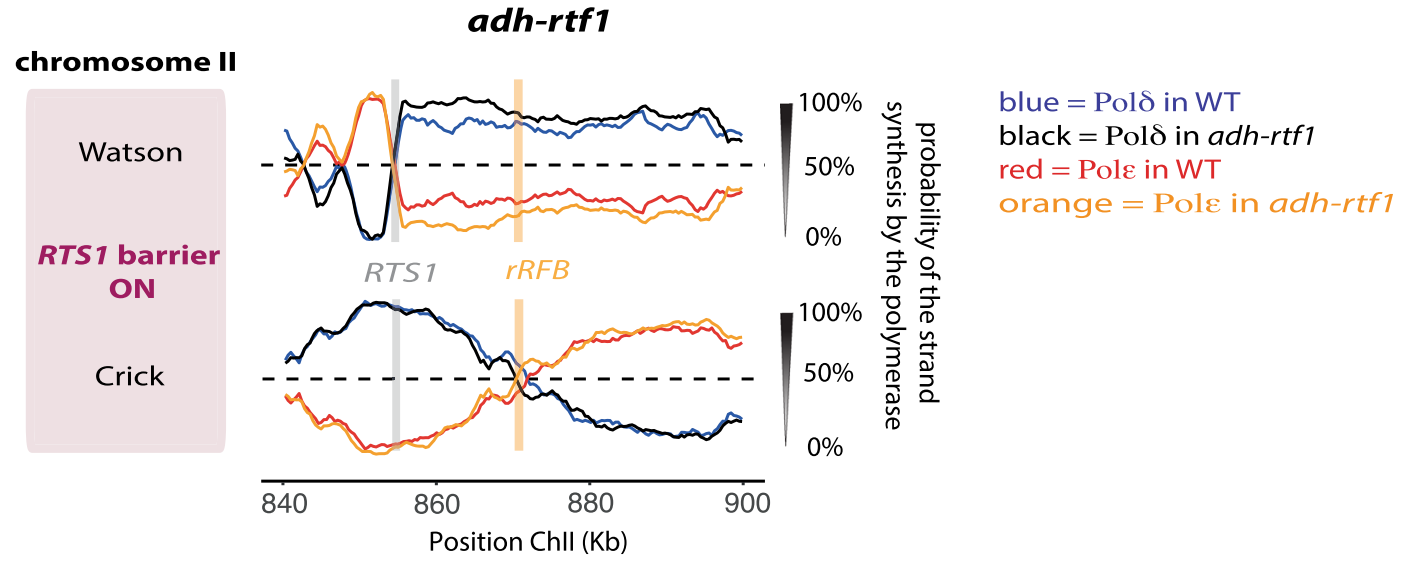

b
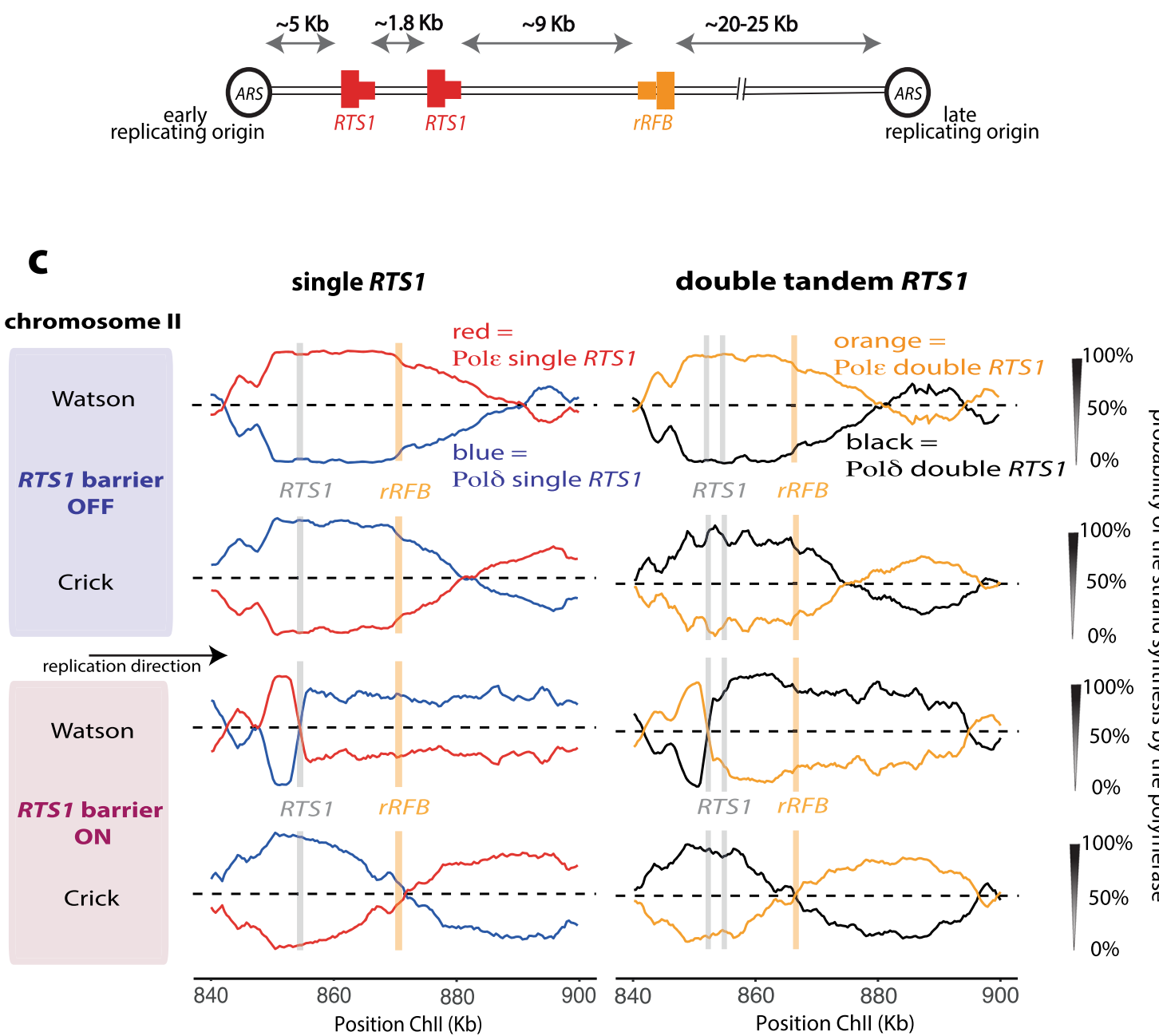

Fig. 3 HR-restarted forks are insensitive to the RTS1 barrier. a Pu-seq traces for the RTS1-rRFB locus are overlaid for two sets of strains: rtf1+ (WT), which has endogenous rtf1 expression (Pol $\delta$ : blue, Pol: $\varepsilon$ red) and adh-rtf1+, where expression is under control of the adh1 promoter (Pol $\delta$ : black, Pol: $\varepsilon$ orange). b Schematic of the tandem RTS1-rRFB locus on chromosome II. The positions of the directional RTS1 and rRFB barriers are shown as red and orange, respectively with the thick bar indicating the directionality of fork arrest. ARS: autonomously replicating sequence. c Pu-seq traces for the tandem RTS1rRFB locus which contains two copies of the RTS1 barrier that are both orientated to arrest left-right forks (right) compared to the original RTS1-rRFB locus (left). Top: barrier off. Bottom: barrier on. 
degrade DNA:RNA hybrids. Preventing RER is essential to the methodology of Pu-seq, but the loss of any DNA:RNA hybrid activity is not required and has the potential to impact on some aspects of replication. A separation of function mutation, $\mathrm{RNH} 2$ $R E D$ has been described and characterised in S. cerevisiae ${ }^{26}$. We created a mutated rnh201 resulting in the equivalent change (Rnh201-P75D:Y245A) and characterised the strain genetically in $S$. pombe to demonstrate successful separation of function (Supplementary Fig. 7).

We performed $\mathrm{Pu}$-seq in the presence or absence of $p k u 70^{+}$in either the rnh201- $d$ or rnh201-RED backgrounds. Genome-wide we observed no significant difference between $r n h 201-d$ and rnh201-RED. We next plotted the traces for both arrest off and arrest on conditions to visualise the RTS1 locus (Fig. 4a). Following arrest and restart we observed that the rnh201-RED mutation resulted in no difference to the traces from either the $p k u 70^{+}$or the $p k u 70-d$ backgrounds. We thus conclude that the specific allele of rnh201 that we use does not influence the experiment.

The presence or absence of Pku70 does not cause a major change in the $\mathrm{Pu}$-seq profiles after fork arrest. However, a careful examination of the data (Fig. 4b) suggests a slight shift in the overall position of termination events (evidenced by the position and slope of Pol $\varepsilon$ usage on the bottom strand) that would be consistent with an increased time for replication restart. The apparent delays are visible in both the rnh2-d and rnh2-RED backgrounds. Using our model of DNA replication, we can estimate that the extent of the delay to HR-restart of forks arrested at RTS1 in the absence of Pku70 as $14 \mathrm{~min}$, which is an increase of $3 \mathrm{~min}(\sim 27 \%)$ when compared to $p k u 70^{+}$background. (Supplementary Fig. 8). These data confirm that $\mathrm{Ku}$ loss has a direct effect on the dynamics of replication fork restart ${ }^{12}$.

One potential explanation for the altered dynamics of replication that we observe in the pku70 mutant is that HRrestart occurs further upstream (left) of the original fork arrest site (see discussion): the increase in resection reported to occur behind the fork in the pku70-d mutant could affect the stability of the leading strand that provides the invading strand during HR and primes HR-restarted replication. If the leading strand were degraded, we would expect to observe strand invasion and thus new $\delta / \delta$ configured DNA synthesis occurring further upstream of the RTS1 sequence. However, in the presence or absence of $\mathrm{Pku} 70$, the transition from $\mathrm{Pol} \varepsilon$ to Pol $\delta$ on the Crick strand, which marks the switch of polymerases, is unchanged (Fig. 4). By modelling various start points of HR-dependent restart we can show that changes in the position of strand invasion of $100 \mathrm{bp}$ would expected to be detected (Supplementary Fig. 9). Thus, we conclude that the leading strand remains equally stable in the $\mathrm{Ku}$ mutant background, despite the increased resection and the delay to restart.

\section{Discussion}

The increased genomic instability that is caused by replication stress when cancer cells are driven into uncoordinated replication by oncogene activation may, in part, be caused by the HRmediated replication restart. To further understand this driver of genetic stability we have applied $\mathrm{Pu}$-seq ${ }^{19}$ to characterise the RTS1 model replication restart system that had previously been established in S. pombe. As expected, our data confirmed the previous conclusions that, when replication is restarted by $\mathrm{HR}$, Pol $\delta$ is used to replicate both the leading and lagging strands ${ }^{10}$. We have also been able to extend several previous observations: we demonstrate that HR-restarted replication forks do not mature into the more canonical $\varepsilon / \delta$ conformation over a distance $\sim 30 \mathrm{~kb}$ and that Pol $\alpha$ is not used at significant levels during HR- restarted replication. This latter observation suggests that the leading and lagging strands are replicated consecutively, rather than being coupled as they are during canonical replication. In support of this we demonstrate that both RPA and potential DNA:RNA hybrids are strongly enriched at the locus that is replicated by HR-restarted replication.

When a replication fork collapses and is unable to resume canonical replication, it can be rescued by a converging fork. However, previous work has shown that a single RTS1 barrier frequently results in HR-dependent replication fork restart even though, in the vast majority of cells, a converging fork would be expected to arrive ${ }^{27}$. This implies that HR-dependent restart occurs irrespective of the status of the locus, but is instead governed by the time it takes to assemble the appropriate machinery. Using GPCR upstream and downstream of RTS1 we have previously estimated that restart occurs after a delay of $\sim 18 \mathrm{~min}$ in the vast majority of cells ${ }^{10}$. A separate study demonstrated that HR protein foci begin to appear $10 \mathrm{~min}$ after the start of $\mathrm{S}$ phase, peak in numbers some $30 \mathrm{~min}$ later and that individual foci can remain present for $>30 \mathrm{~min}^{27}$. To reconcile these two data sets, we have speculated that HR-mediated restart occurs in most cells after a delay of $\sim 18 \mathrm{~min}$ and that $\mathrm{HR}$ proteins remain associated with the locus during HR-restarted replication progress (and possibly during fork convergence and termination with the incoming canonical fork).

The utility of combining a physical profile of replication dynamics with a mathematical model of DNA replication that can incorporate complementary genetic and physical data is exemplified by our consideration of the duration of replication arrest at RTS1. By extending a previously reported Monte Carlo simulation of $S$. pombe DNA replication ${ }^{18}$ to include the ability to modify replication speed in a time and location-dependent manner (see Materials and Methods section) we observed that the best fit of the model to the experimental data (given by the Euclidean distance) is a delay of $11 \mathrm{~min}$ at RTS1, before replication is restarted by HR. This estimate is quite distinct from the observed experimental data obtained by qPCR of $18 \mathrm{~min}^{10}$. A potential explanation for this discrepancy is that the timing estimate assumes that the speed of HR-restarted replication is equivalent to the speed of the canonical fork (which we modelled at $1.8 \mathrm{~kb} / \mathrm{min}$ ). However, in $S$. cerevisiae, it has been estimated that break-induced replication, which proceeds via a migrating $\mathrm{D}$ loop, progresses at between 3 and $4 \mathrm{~kb}$ a minute ${ }^{28}$. If we increase the speed of the HR-restarted fork to $3 \mathrm{~kb}$ in the model, then our estimate of the delay to DNA replication at RTS1 is extended to $17.5 \mathrm{~min}$. Thus, based on the qPCR evidence of replication time upstream and downstream of RTS1 varying by $18 \mathrm{~min}$ when the barrier is active, we propose that HR-dependent replication restarts relatively synchronously and proceeds $\sim 50 \%$ faster than canonical replication.

When a fork is restarted by HR following arrest at the RTS1 barrier, it clearly is able to progress through the barrier. We have previously speculated that the restarted replication apparatus is distinct from the canonical replisome and that the HR-dependent apparatus is not sensitive to the barrier ${ }^{15}$. Here we have tested this by following the dynamics of HR-restarted forks as they pass through a second copy of RTS1. Visual examination of the data suggests no appreciable evidence of a delay to the HR-restart replication imposed by the second barrier. By modelling a variety of scenarios whereby HR-restarted forks are delayed between 0 and $30 \mathrm{~min}$ at the second barrier, we demonstrate that the best fit of the experimental data to the model is $0 \mathrm{~min}$ delay. This demonstrates that HR-restarted replication is insensitive to at least this obstacle, which efficiently arrests canonical forks. Thus, the distinct nature of the restarted apparatus may provide cells with an alternative replication machine that is able to pass some 
rnh2014 + rnh201-RED

a

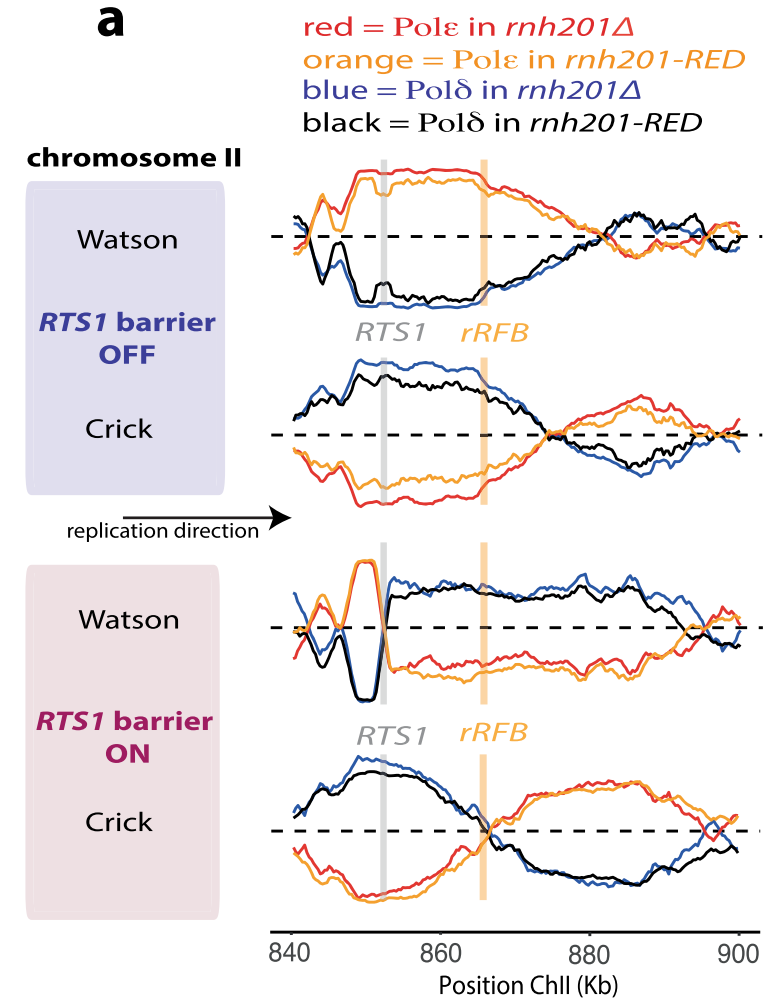

pKu704 + WT in rnh2014

b $\quad$ red $=$ Pole in WT

orange $=$ Pole in $p K u>0 \triangle$

blue $=$ Pols in WT

chromosome II

black $=$ Pold in $p K u 70 \triangle$

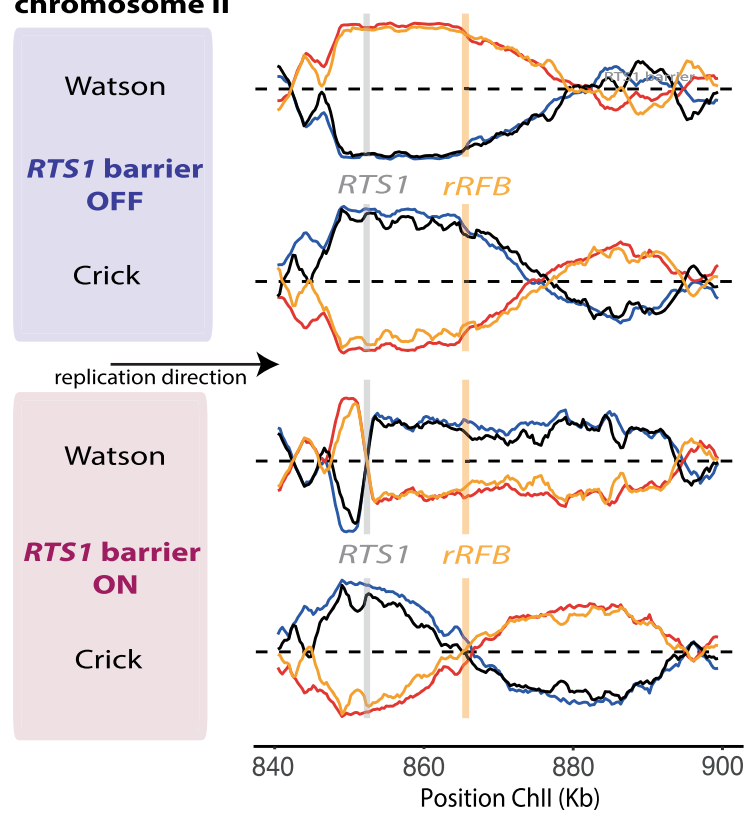

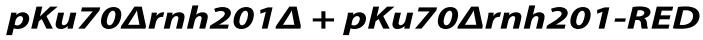

red $=$ Pole in $p K u 70 \triangle r n h 201 \Delta$

orange $=$ Pole in $p K u 70 \Delta r n h 201-R E D$

blue $=$ Pols in $p K u 70 \Delta r n h 201 \Delta$

black $=$ Pold in pKu70 $r$ rnh201-RED
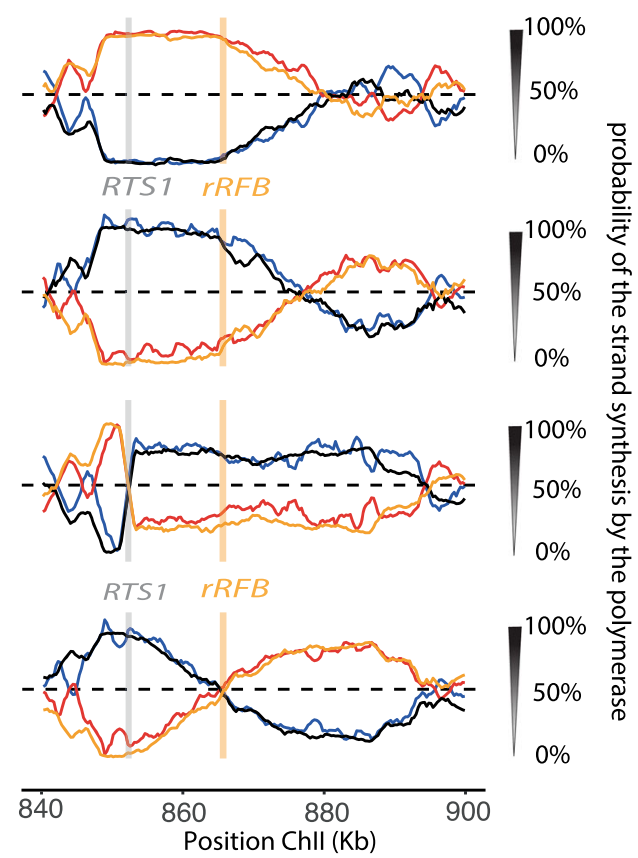

pKu704 + WT in rnh201-RED

red $=$ Pole in WT

orange $=$ Pole in $p K u>O \triangle$

blue $=$ Pols in WT

black $=$ Pold in $p K u>0 \triangle$
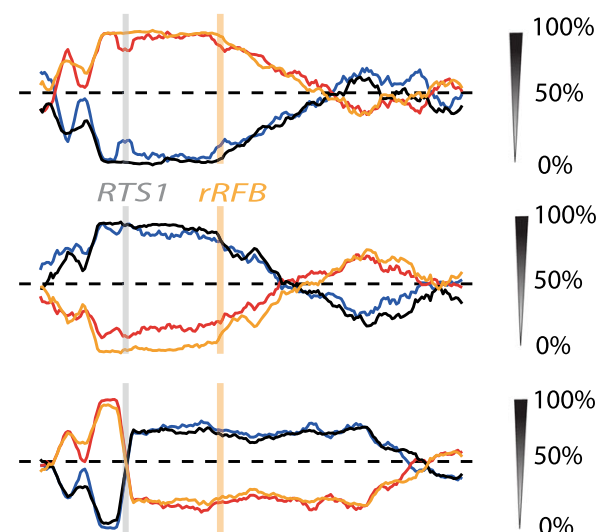

RTS1 rRFB

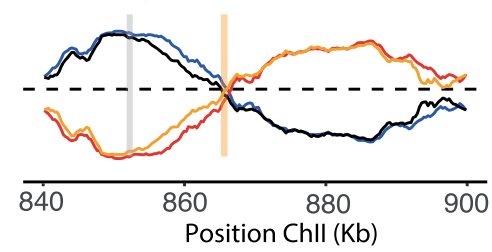

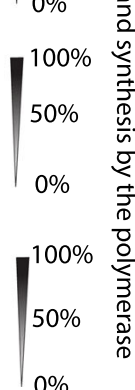

Fig. 4 Loss of pku70 ${ }^{+}$results does not destabilise the leading stand primer. a Overlays of Pu-seq traces of the RTS1-rRFB locus either with or without arrest and in the presence and absence of $p k u 70^{+}$. Left: in the rnh201-d background. Right: in the rnh201-RED background. b For the purpose of comparison, overlays of the Pu-seq traces for rnh201-d and rnh201-RED backgrounds. Left: in pku70+. Right: in pku70-d. 


\section{Homologous recombination- dependent restart}

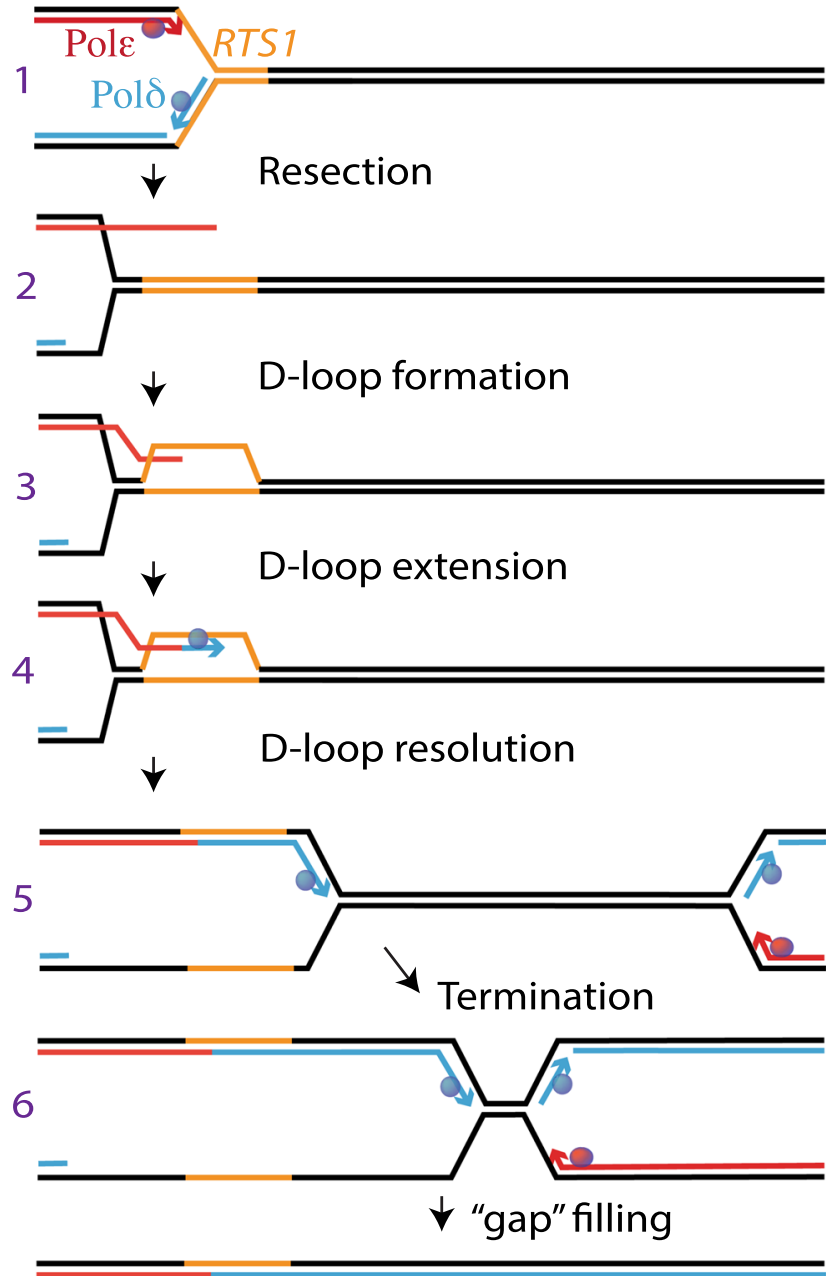

7

Fig. 5 Schematic of HR-dependent restart. (1) Canonical RF arrested at RTS1. DNA synthesised by Pol $\varepsilon$ is shown in red. DNA synthesised by Pol $\delta$ shown in blue. Template: black. RTS1 sequence: orange. (2) Resection generates a free $3^{\prime}$ end. (3) Recombination factors generate a D-loop at the site of stalling. (4) The D-loop primer is extended, most likely by Pol $\delta$. The D-loop is resolved, establishing semi-conservative DNA synthesis. (5). Pol $\delta$, with a minimal contribution from Pol $\alpha$, synthesises the leading strand. DNA synthesis is insensitive to further downstream RTS1 barriers. (6) Termination with the incoming canonical fork. (7). The gap is filled by Pol $\delta$.

barriers more effectively. Data from $S$. cerevisiae indicate that during break-induced replication (BIR), a homologous recombination-dependent replication initiation event that is occurs in response to a persistent double strand break in G2 arrested cells (where replication subsequently proceeds as a migrating D-loop), strand unwinding is dependent on helicases other than the canonical Cdc45/MCM2-7/GINS (CMG) replicative helicase ${ }^{29}$. We speculate that CMG is not involved in the restart or subsequent $\delta / \delta$ replication we observe here and that alternative helicases, such as $\mathrm{Pfh} 1^{29}$, are involved. These would potentially be insensitive to the RTS1 barrier.

The CMG helicase is important for replication fidelity. The regulation of CMG loading onto DNA is also strictly regulated to prevent erroneous initiation and thus re-replication ${ }^{30}$. If replication that is restarted by HR employs alternative helicases as we propose, this is likely because the loading of CMG in this context would compromise the strict regulation of CMG loading. However, the use of alternatives to the CMG comes at a cost - the HR restarted fork is highly error prone and, since we also provide evidence that leading and lagging strand synthesis are not coupled, significant quantities of ssDNA will be present that would be sensitive to oxidative stress ${ }^{31}$. In the yeast model systems there is no equivalent to the MCM8/9 helicase ${ }^{32}$ that is proposed to function in metazoan cells during HR-dependent tolerance of replication stress ${ }^{33}$. It is possible that the increased errors that occur when replication is restarted by HR may have been a driving force behind the evolution of the MCM8/9 helicase.

Having established a system to track replication dynamics we were interested to explore the effects of deregulating resection by deletion of pku70. A recent study demonstrated that loss of Ku caused the loss of Rad50- and Ctp1-dependent two-stage resection regulation and resulted in extended tracts of ssDNA that stretch several kilobases behind the arrested fork ${ }^{12}$. By measuring the error rate in a replication slippage assay, the authors suggested that, because the error rate decreased by $\sim 50 \%$ and this could be rescued by delaying the incoming fork, this could be interpreted as a delay to HR-dependent restart. The most prosaic expectation would predict that the loss of the requirement that Ku70 to be removed from a reversed fork by MRN would actually lead to faster restart. We considered an alternative explanation for the delay: that $3^{\prime}$ end which primes the strand invasion event is unstable and, during the extended $5^{\prime}-3^{\prime}$ exonuclease activity, it is shortened. This would result in replication restart occurring further upstream from the initial arrest site. Even if the restart occurred at the normal time (i.e. after $\sim 18$ minus), this would manifest as a delay, since the HR-restarted fork would have further to travel and thus an increased proportion of the locus would be replicated by the converging canonical fork.

Using our Pu-seq assay we were able to demonstrate that the transition from Pol $\varepsilon$ to Pol $\delta$ occurs at the same position in $p k u 70^{+}$and $p k u 70-d$ backgrounds. Since the position of this transition is caused by the switch from Pol $\varepsilon$ during canonical replication to Pol $\delta$ when replication is restarted by $\mathrm{HR}$, this demonstrates that the $3^{\prime}$ end maintains the same stability even when resection is deregulated by loss of $p k u 70$ and that restart occurs at the same place irrespective of $\mathrm{Ku}$ status. Thus, any processing of the $3^{\prime}$ end is not influenced by $\mathrm{Ku}$ or the extent of $5^{\prime}$ end resection. By modelling scenarios where replication restart is shifted we were able to confirm that a movement of 100 bases should be detectable at the resolution of the experiment. By modelling the kinetics of replication, we were able to confirm that loss of Pku70 indeed resulted in an additional delay to replication restart of $3 \mathrm{~min}$ (an additional 27\% compared to $p k u 70^{+}$). This manifests as a shift in the positions of termination of the HRrestarted forks with converging canonical forks.

It could be asked why a $27 \%$ increase in the time taken to restart DNA replication in the absence of $\mathrm{Ku}$ changes the mutation frequency immediately downstream of the RTS1 barrier by a factor of two ${ }^{12}$. It should be noted that these genetic experiments were performed using the RTS1 barrier integrated at the ura 4 locus on chromosome III. At this locus, as opposed to our optimised ChrII locus, there are more forks converging on the arrest site from active downstream origins and thus a modest delay to restart would be expected to have a significantly more pronounced effect on the percentage of DNA downstream of the barrier that is replicated by an HR-restarted (error prone) fork as opposed to a converging canonical fork. It remains unclear why there is a delay to restart in the pku70- $d$ background, although it is proposed to be linked to the reduction of RPA and Rad51 observed by microscopy ${ }^{12}$. 
HR-restarted replication is likely to contribute to genome instability, particularly in cancer cells experiencing intrinsic replication stress. While canonical replication forks are remarkably accurate, HR restarted replication is highly error prone. This is likely directly linked to the non-canonical nature of the restarted fork (Fig. 5): restarted replication proceeds without coupling between the leading and lagging strands resulting in long tracts of single-stranded DNA and both strands are synthesised by Pol $\delta$ without any apparent maturation to a more canonical $\delta / \varepsilon$ configuration. Our analysis also implies that the CMG is not the helicase for restarted forks. This may have a benefit in allowing the fork to bypass obstacles that would otherwise impede the canonical helicase.

\section{Methods}

Strain construction. To introduce the RTS1 sequence into 4 loci analysed in Supplementary Fig. 1, the kan cassette from pFAX-kanMX6 was amplified by the following primers: 461/462 (mns1 locus), 463/464 (tel2 locus), 465/466 (mek1 locus), and F3/R4 (spo9 locus). PCR amplified fragments (which introduce a loxP and a loxM site flanking the kan cassette) were transformed into strain 503 to form strains $1144,1146,1148$ and KA2 respectively. These strains were subsequently transformed with pAW8-RTS1-ura-2/3ter ${ }^{10}$ to integrate a construct between the lox sites containing RTS1-ura-2/3ter. This resulted in strains $1158,1162,1166$, KA7

To create the BAY4 strain used to analyse the effect of the rRFB (Supplementary Fig. 2) strain 1144 (containing loxP- loxM flanking the kan cassette on ChrI) was transformed with pAW8-10xrRFB-ura to integrate 10xRFB-ura between the lox sites.

To create the optimised ChrII locus (Fig. 1) the 10xrRFB sequence was integrated downstream of RTS1 barrier. Primers F29 and R30 were used to amplify $\mathrm{ura}^{+}$and the product was transformed into KA2 and checked for insertion onto the ChrII between SPBC36.10.1 and SPBC36.11.1 to form strain KA39. The $\mathrm{ura}^{+}$gene was then replaced by $10 x \mathrm{rRFB}$ using a fragment amplified from pAW8-10xrRFB using primers F41 and R42 to form strain KA56. KA56 was transformed by pAW8-RTS-ura including loxP and loxM sites. This created the strain KA58.

To construct the inverted RTS1 locus used in Fig. 2, primers F229 and R230 were used to amplify $\mathrm{ura}^{+}$and the product was transformed into KA2 and checked for insertion onto the ChrII between $p w p 2$ and $w d r 83$ to form strain KA218. The $u r a 4^{+}$gene was then replaced by RTS1 using a fragment amplified with primers F241 and R242 to form strain KA233. KA233 was transformed by pAW8-RTS-ura including loxP and loxM sites. The kan cassette between spo9 and clr6 on ChrII close to early firing origin was replaced by the RTS1 construct. This created the strain KA237.

To construct the tandem RTS1 barrier used in Fig. 3 we modified pAW8-RTSura to inserted a second RTS1 downstream of ura $^{+}$between the SacI-SalI sites. The plasmid was transformed into KA56 which contains the kan cassette flanked by loxP and LoxM sites. Cre-lox recombination replaced the kan cassette by the RTS-ura-RTS cassette between spo 9 and $c l r 6$ on ChrII, to give strain KA101.

To construct rnh201-RED mutant allele the S. cerevisiae Rnh201-RED mutant sequence ${ }^{26}$ was aligned to $S$. pombe Rnh201. S. cerevisiae Rnh201 P45D and Y219A changes corresponded to $S$. pombe Rnh201 P75D and Y245A (Fig. S1A). The $S$. pombe rnh201-P75D-Y245A gene sequence was synthesised (integrated DNA technologies) and cloned into Cre-expression vector pAW $8^{34}$ to create rnh201RED-pAW8. The hphMX6, natMX6 and kanMX6 antibiotic resistance markers ${ }^{35}$ were amplified using primers 264 and 265 and cloned into rnh201-RED-pAW8 to create rnh201-RED-hphMX6-pAW8, rnh201-RED-natMX6-pAW8 and rnh201RED-kanMX6-pAW8 respectively. All constructs were confirmed by sequencing The rnh201-RED-hphMX6-pAW8, rnh201-RED-natMX6-pAW8 and rnh201RED-kanMX6-pAW8 plasmids were used to transform S. pombe. 'rnh201 base strain' AW1480 and the rnh201-RED-MX6 sequences integrated by recombinasemediated cassette exchange ${ }^{34}$. This created strains AW1563 (rnh201-RED: hphMX6), AW1565 (rnh201-RED:natMX6) and AW1566 (rnh201-RED:kanMX6).

To test rNTP incorporation rates, strain AW1563 (rnh201-RED:hphMX6) was crossed to strain AW1227 (cdc20M630F) to create strain AW1232 (rnh201-RED: kanMX6, cdc20M630F). Control strains included $r n h 201^{+}$, AW1466 (cdc20$M 630 F)$ and AW1405 ( $r n h 201 \Delta, c d c 20-M 630 F)$. Approximately $3 \times 10^{8}$ logarithmically growing cells were collected and the genomic DNA extracted ${ }^{36}$ and incubated in the presence of $0.3 \mathrm{~N} \mathrm{KOH}$ for $1 \mathrm{~h}$ at $55^{\circ} \mathrm{C}$. The alkali was then neutralised by the addition of equimolar amount of $\mathrm{HCl}$ and the reaction mix isopropanol precipitated. A portion of the genomic DNA was untreated to confirm the integrity of the DNA extract. Following 2\% agarose/1xTBE gel electrophoresis and staining with acrydine orange (Invitrogen), the degree of fragmentation observed was compared relative to the non-treated DNA control.

To perform spot assays of S. pombe strains AW278 (WT), AW1229/AW1230 (rnh201-RED, rnh1-d top1-d) and AW1198/AW1199 (rnh201-d, rnh1-d and top1d) were serially diluted 10 -fold in water and spotted on YEA plates.

A list of strains and associated genotypes is given in Supplementary Table 1.
Cell synchronisation. Cells were synchronised using the $c d c 2-a s M 17$ allele ${ }^{37}$ and 3-Br-PP1 (2uM final, Abcam) at $28^{\circ} \mathrm{C}$ for $3 \mathrm{~h}$. Upon the removal of 3-Br-PP1, cells were grown at $30^{\circ} \mathrm{C}$ and samples collected at the indicated times.

ChIP-qPCR:

In all, $40 \mathrm{ml}$ of log-phase cells were grown in YES or EMM liquid media at $30^{\circ} \mathrm{C}$ and crosslinked in $1 \%$ formaldehyde $\left(15 \mathrm{~min} \mathrm{RT}^{\circ}\right)$ followed by addition of $5 \mathrm{ml} 2.5$ $\mathrm{M}$ glycine for $5 \mathrm{~min}$. Cells were centrifuged $(4000 \times g, 5 \mathrm{~min})$ and washed with 10 $\mathrm{ml}$ PBS and the pellet frozen. For RNA:DNA hybrid ChIP cells were not crosslinked but NaN3 (0.1\% final) was added.

Cell pellets were resuspended in $400 \mu \mathrm{l}$ ChIP lysis buffer (50 mM HEPES pH 7.4; $140 \mathrm{mM} \mathrm{NaCl} ; 1 \%$ Triton X100; 0.1\% NaDeoxycholate; protease inhibitors), disrupted by glass bead beating using a FastPrep-24 (MPbiomedical) homogenizer. Homogonised material was harvested by 1 -minute centrifugation to remove glass beads, washed once with $1 \mathrm{ml} \mathrm{ChIP} \mathrm{lysis} \mathrm{buffer} \mathrm{and} \mathrm{resuspended} \mathrm{in} 350 \mathrm{ml}$ of ChIP lysis buffer. The chromatin DNA was sheared by a Qsonica ultrasonicator to the size range of $\sim 500 \mathrm{bp}$. Samples were centrifuged for $1 \mathrm{~min}$ and the supernatant incubated with antibody (GFP polyclonal antibody from Invitrogen, cat.no. A11122, dilution 1:150) or S9.6 (Sigma-Aldrich, cat.no. MABE1095, dilution 1:200) for $1 \mathrm{~h}$ at $4^{\circ} \mathrm{C}$. Protein $\mathrm{G}$ coupled Dynabeads (Thermo Fisher) were used for immunoprecipitation overnight at $4{ }^{\circ} \mathrm{C}$. The beads were washed twice for $5 \mathrm{~min}$ at 4 ${ }^{\circ} \mathrm{C}$ in $1 \mathrm{ml}$ of each of the following buffers: ChIP lysis buffer, high salt lysis buffer (50 mM HEPES pH 7.4; $500 \mathrm{mM} \mathrm{NaCl;1 \%} \mathrm{Triton} \mathrm{X100;} \mathrm{0.1 \%} \mathrm{NaDeoxycholate)}$ and wash buffer $(10 \mathrm{mM}$ Tris $\mathrm{pH} 8.0 ; 250 \mathrm{mM} \mathrm{LiCl} ; 0.5 \%$ NP-40; $0.5 \%$ NaDeoxycholate; $1 \mathrm{mM}$ EDTA) and once in TE buffer (10 mM Tris pH 8.0, $1 \mathrm{mM}$ EDTA) for a minute. Beads were resuspended in (110 ul) elution buffer (50 mM Tris pH $8.0 ; 1 \%$ SDS; 10 mM EDTA) and $3 \mu \mathrm{l}$ cocktail of Rnases (Invitrogen) was added and incubated at $30^{\circ} \mathrm{C}$ for $2 \mathrm{~h}$ with shaking. In all, $5 \mu \mathrm{l}$ of $20 \mathrm{mg} / \mathrm{ml}$ Proteinase $\mathrm{K}$ (Sigma Aldrich) was added and incubated at $65^{\circ} \mathrm{C}$ for $2 \mathrm{~h}$ with shaking. The samples were purified using a PCR purification kit (Quiagen) and analysed by qPCR using LUNA qPCR mix (NEB) on a real-time detection system (Agilent). Fold enrichment was calculated as ChIP/Input. Primers used for qPCR are listed in Supplementary Table 2. The Chip data are available in Supplementary Data 1.

Polymerase usage sequencing. The published protocol ${ }^{19}$ was used with minor modifications: size selection was performed using a Blue Pippin (Sage Science). In some instances we used $r n h 201-R E D$ instead of $r n h 201:: k a n$ (see results). Sequence files were aligned with Bowtie 2 and alignment data converted to counts with custom Perl script ${ }^{19}$. Analysis of polymerase usage was performed with custom $\mathrm{R}$ script $^{19}$. Since Pol $\alpha$ is relatively less used than Pol $\delta$ or Pol $\varepsilon$, the overplayed data is multiplied by 10 to match the approximate scale of the other polymerases. We do not know the relative rNTP incorporation rates of the three polymerases and have not made an assumption on the relative contribution of $\mathrm{Pol} \alpha$ to overall replication.

Mathematical modelling. We simulated the replication process using a variation of the model presented by Kelly and Callegari ${ }^{18}$. We extended the model to include time- and location-dependent fork velocity to allow the modelling of the replication barriers. The model determines origin locations and efficiency based on the AT-richness and transcription activity. We optimised the parameters of the model to globally fit the wild-type replication profile, using the line search approach akin to the methodology of Kelly and Callegari. We suppressed some minor origins (which Fig. 2 demonstrates are not used) in the region of interest to fit to the traces of polymerase usage in the wild type strain. These origins were otherwise present in the simulation but not in acquired data by $\mathrm{Pu}$-seq.

We modelled the fork barrier by means of the fork velocity: at the location of the barrier, the modelled fork velocity drops to zero for some time, and then increases to the original value. We use a constant velocity of $1800 \mathrm{~kb} / \mathrm{min}$ for the rest of the chromosome. We defined separately the velocity of left- and righttravelling forks in order to model unidirectional fork barriers.

We used the Euclidean norm of the difference between model solution and experimental data to quantify the model error. Where appropriate a Savitzky-Golay filter was used to smooth the line. In all the simulations we used an ensemble of 1000 cells, and then we validated the optimal result by further simulating an ensemble of 10,000 cells

All the quantities that we optimised using the model were found by performing a line search. We used this approach to find the model predictions for the fraction of forks arrested at the RTS1 barrier, the time to restart for arrested forks and the effect of the RTS1 barrier on restarted forks.

Reporting summary. Further information on research design is available in the Nature Research Reporting Summary linked to this article.

\section{Data availability}

The datasets generated during the current study are available in the NCBI GEO repository under accession number GSE153731. The data supporting the findings of this study are available from the corresponding authors upon reasonable request.

\section{Code availability}

The code for the model used is available at https://doi.org/10.5281/zenodo.4423130 
Received: 23 July 2020; Accepted: 16 January 2021;

Published online: 10 February 2021

\section{References}

1. Drake, J. W., Charlesworth, B., Charlesworth, D. \& Crow, J. F. Rates of spontaneous mutation. Genetics 148, 1667-1686 (1998).

2. Chen, L. et al. CNV instability associated with DNA replication dynamics: evidence for replicative mechanisms in CNV mutagenesis. Hum. Mol. Genet 24, 1574-1583 (2015).

3. López Castel, A., Cleary, J. D. \& Pearson, C. E. Repeat instability as the basis for human diseases and as a potential target for therapy. Nat. Rev. Mol. Cell Biol. 11, 165-170 (2010).

4. Magdalou, I., Lopez, B. S., Pasero, P. \& Lambert, S. A. The causes of replication stress and their consequences on genome stability and cell fate. Semin. Cell Dev. Biol. 30, 154-164 (2014).

5. Kawabata, T. et al. Stalled fork rescue via dormant replication origins in unchallenged $\mathrm{S}$ phase promotes proper chromosome segregation and tumor suppression. Mol. Cell 41, 543-553 (2011).

6. Ozeri-Galai, E. et al. Failure of origin activation in response to fork stalling leads to chromosomal instability at fragile sites. Mol. Cell 43, 122-131 (2011).

7. Lambert, S. \& Carr, A. M. Replication stress and genome rearrangements: lessons from yeast models. Curr. Opin. Genet. Dev. 23, 132-139 (2013)

8. Mayle, R. et al. DNA REPAIR. Mus81 and converging forks limit the mutagenicity of replication fork breakage. Science 349, 742-747 (2015).

9. Ait Saada, A. et al. Unprotected replication forks are converted into mitotic sister chromatid bridges. Mol. Cell 66, 398-410 (2017).

10. Miyabe, I. et al. Polymerase $\delta$ replicates both strands after homologous recombination-dependent fork restart. Nat. Struct. Mol. Biol. 22, 932-938 (2015).

11. Mizuno, K., Lambert, S., Baldacci, G., Murray, J. M. \& Carr, A. M. Nearby inverted repeats fuse to generate acentric and dicentric palindromic chromosomes by a replication template exchange mechanism. Genes Dev. 23, 2876-2886 (2009).

12. Teixeira-Silva, A. et al. The end-joining factor $\mathrm{Ku}$ acts in the end-resection of double strand break-free arrested replication forks. Nat. Commun. 8, 1982 (2017).

13. Iraqui, I. et al. Recovery of arrested replication forks by homologous recombination is error-prone. PLoS Genet. 8, e1002976 (2012).

14. Jalan, M., Oehler, J., Morrow, C. A., Osman, F. \& Whitby, M. C. Factors affecting template switch recombination associated with restarted DNA replication. Elife 8, e41697 (2019).

15. Mizuno, K., Miyabe, I., Schalbetter, S. A., Carr, A. M. \& Murray, J. M. Recombination-restarted replication makes inverted chromosome fusions at inverted repeats. Nature 493, 246-249 (2013).

16. Donnianni, R. A. \& Symington, L. S. Break-induced replication occurs by conservative DNA synthesis. Proc. Natl Acad. Sci. USA 110, 13475-13480 (2013).

17. Daigaku, Y. et al. A global profile of replicative polymerase usage. Nat. Struct. Mol. Biol. 22, 192-198 (2015).

18. Kelly, T. \& Callegari, A. J. Dynamics of DNA replication in a eukaryotic cell. Proc. Natl Acad. Sci. USA 116, 4973-4982 (2019)

19. Keszthelyi, A., Daigaku, Y., Ptasińska, K., Miyabe, I. \& Carr, A. M. Mapping ribonucleotides in genomic DNA and exploring replication dynamics by polymerase usage sequencing ( $\mathrm{Pu}-\mathrm{seq})$. Nat. Protoc. 10, 1786-1801 (2015).

20. Dalgaard, J. Z. \& Klar, A. J. A DNA replication-arrest site RTS1 regulates imprinting by determining the direction of replication at mat 1 in $\mathrm{S}$. pombe. Genes Dev. 15, 2060-2068 (2001).

21. Krings, G. \& Bastia, D. swil- and swi3-dependent and independent replication fork arrest at the ribosomal DNA of Schizosaccharomyces pombe. Proc. Natl Acad. Sci. USA 101, 14085-14090 (2004).

22. Calzada, A., Hodgson, B., Kanemaki, M., Bueno, A. \& Labib, K. Molecular anatomy and regulation of a stable replisome at a paused eukaryotic DNA replication fork. Genes Dev. 19, 1905-1919 (2005).

23. Bastia, D. et al. Phosphorylation of CMG helicase and Tofl is required for programmed fork arrest. Proc. Natl Acad. Sci. USA 113, E3639-E3648 (2016).

24. Ohle, C. et al. Transient RNA-DNA hybrids are required for efficient doublestrand break repair. Cell 167, 1001-1013 (2016).

25. Sparks, J. L. et al. RNase H2-initiated ribonucleotide excision repair. Mol. Cell 47, 980-986 (2012)

26. Chon, $\mathrm{H}$. et al. RNase $\mathrm{H} 2$ roles in genome integrity revealed by unlinking its activities. Nucleic Acids Res. 41, 3130-3143 (2013).
27. Nguyen, M. O., Jalan, M., Morrow, C. A., Osman, F. \& Whitby, M. C. Recombination occurs within minutes of replication blockage by RTS1 producing restarted forks that are prone to collapse. Elife 4, e04539 (2015).

28. Malkova, A., Naylor, M. L., Yamaguchi, M., Ira, G. \& Haber, J. E. RAD51dependent break-induced replication differs in kinetics and checkpoint responses from RAD51-mediated gene conversion. Mol. Cell Biol. 25, 933-944 (2005).

29. Wilson, M. A. et al. Pif1 helicase and Pol $\delta$ promote recombination-coupled DNA synthesis via bubble migration. Nature 502, 393-396 (2013).

30. Blow, J. J. \& Dutta, A. Preventing re-replication of chromosomal DNA. Nat Rev. Mol. Cell Biol. 6, 476-486 (2005).

31. Sakofsky, C. J. et al. Break-induced replication is a source of mutation clusters underlying kataegis. Cell Rep. 7, 1640-1648 (2014).

32. Griffin, W. C. \& Trakselis, M. A. The MCM8/9 complex: a recent recruit to the roster of helicases involved in genome maintenance. DNA Repair 76, 1-10 (2019).

33. Natsume, T. et al. Acute inactivation of the replicative helicase in human cells triggers MCM8-9-dependent DNA synthesis. Genes Dev. 31, 816-829 (2017)

34. Watson, A. T., Garcia, V., Bone, N., Carr, A. M. \& Armstrong, J. Gene tagging and gene replacement using recombinase-mediated cassette exchange in Schizosaccharomyces pombe. Gene 407, 63-74 (2008).

35. Hentges, P., Van Driessche, B., Tafforeau, L., Vandenhaute, J. \& Carr, A. M. Three novel antibiotic marker cassettes for gene disruption and marker switching in Schizosaccharomyces pombe. Yeast 22, 1013-1019 (2005).

36. Watson, A. T., Werler, P. \& Carr, A. M. Regulation of gene expression at the fission yeast Schizosaccharomyces pombe urg1 locus. Gene 484, 75-85 (2011).

37. Aoi, Y., Kawashima, S. A., Simanis, V., Yamamoto, M. \& Sato, M. Optimization of the analogue-sensitive $\mathrm{Cdc} 2 / \mathrm{Cdk} 1$ mutant by in vivo selection eliminates physiological limitations to its use in cell cycle analysis. Open Biol. 4, 140063 (2014)

\section{Acknowledgements}

This work was funded by the Wellcome Trust. Grant number: 110047/Z/15/Z. KN acknowledges Marie Skłodowska-Curie Fellowship 749631-Rep_Fork Restart

\section{Author contributions}

K.N., E.C-F. and A.M.C. conceived and designed the study. K.N., A.T.W., A.B. and I.M acquired the data, K.N., E.C-F. and A.M.C. interpreted the data. K.N. and A.M.C. drafted the manuscript. All authors edited the manuscript

\section{Competing interests}

The authors declare no competing interests.

\section{Additional information}

Supplementary information The online version contains supplementary material available at https://doi.org/10.1038/s41467-021-21198-0.

Correspondence and requests for materials should be addressed to K.N. or A.M.C.

Peer review information Nature Communications thanks Thomas Kunkel and other, anonymous reviewers for their contributions to the peer review of this work.

Reprints and permission information is available at http://www.nature.com/reprints

Publisher's note Springer Nature remains neutral with regard to jurisdictional claims in published maps and institutional affiliations.

\footnotetext{
(c) (i) Open Access This article is licensed under a Creative Commons Attribution 4.0 International License, which permits use, sharing adaptation, distribution and reproduction in any medium or format, as long as you give appropriate credit to the original author(s) and the source, provide a link to the Creative Commons license, and indicate if changes were made. The images or other third party material in this article are included in the article's Creative Commons license, unles indicated otherwise in a credit line to the material. If material is not included in the article's Creative Commons license and your intended use is not permitted by statutory regulation or exceeds the permitted use, you will need to obtain permission directly from the copyright holder. To view a copy of this license, visit http://creativecommons.org/ licenses/by/4.0/.
}

(C) The Author(s) 2021 